\title{
Different Hypercoagulable Profiles in Patients with COVID-19 Admitted to the Internal Medicine Ward and the Intensive Care Unit
}

\author{
Annalisa Boscolo ${ }^{1, *}$ Luca Spiezia ${ }^{2, *}$ Christelle Correale ${ }^{3}$ Nicolò Sella $^{3}$ Elisa Pesenti ${ }^{3}$ \\ Luca Beghetto $^{3}$ Elena Campello ${ }^{4}$ Francesco Poletto $^{2}$ Lorenzo Cerruti ${ }^{2}$ Marco Cola $^{5}$ \\ Alessandro De Cassai ${ }^{1}$ Laura Pasin ${ }^{1}$ Serra Eugenio ${ }^{1}$ Roberto Vettor ${ }^{4}$ Anna Maria Cattelan ${ }^{5}$ \\ Paolo Simioni ${ }^{2}$ Paolo Navalesi ${ }^{1,3}$
}

${ }^{1}$ Anaesthesia and Intensive Care Unit, Padua University Hospital,
Padua, Italy
${ }^{2}$ General Medicine and Thrombotic and Hemorrhagic Diseases Unit,
Department of Medicine, Padua University Hospital, Padua, Italy
${ }^{3}$ Department of Medicine (DIMED), Padua University Hospital, Padua, Italy
${ }^{4}$ Division of Internal Medicine, Department of Medicine, Padua
University Hospital, Padua, Italy
${ }^{5}$ Infectious Disease Unit, Padua University Hospital, Padua, Italy

Thromb Haemost 2020;120:1474-1477.

Coagulation abnormalities were common findings in critically ill patients affected by coronavirus infection disease (COVID)19 and often correlated to more severe illness and poor prognosis. ${ }^{1-3}$ We recently used Rotational Thromboelastometry (ROTEM) to describe severe hypercoagulable profiles and high incidence of symptomatic venous thromboembolic (VTE) events in a small group of COVID-19 patients with acute respiratory failure admitted to intensive care unit (ICU). ${ }^{4}$ However, it remains unclear whether COVID-19 patients with mild clinical symptoms, admitted to internal medicine wards (IMWs), present with different coagulation profiles.

The primary outcome of this prospective observational study was to investigate the difference in maximum clot firmness (MCF) between IMW and ICU patients, which reflects the extent of hypercoagulability. Secondary outcome was the incidence of symptomatic VTE.

The protocol was approved by the local institutional ethical committee and all consecutive patients admitted to ICU (between February 28 and April 7, 2020) and to IMW (between March 27 and April 14, 2020) for acute respiratory failure caused by SARS-CoV-2 infection were considered for enrollment (-Supplementary Material, -Fig. S1, online only). Exclusion criteria were: pregnancy, preexisting bleeding or hematological disorders, acquired coagulopathies, $<18$ or $>80$ years of age, Child's C liver disease,

* These authors equally contributed to this work.

received

May 14, 2020

accepted after revision

June 8,2020
Address for correspondence Annalisa Boscolo, MD, PhD, Anaesthesia and Intensive Care Unit, Padua University Hospital, 13 Gallucci St., 35121 Padova, Italy (e-mail: annalisa.boscolo@gmail.com).

severe chronic kidney disease, active cancer, and ongoing anticoagulant or antiplatelet therapies.

The following EXTEM, INTEM, and FIBTEM parameters were measured: clotting time (CT, second); clot formation time (CFT, second); MCF (mm); and ThromboDynamic Index (TDI), the ratio between MCF/(CT $+\mathrm{CFT}) .{ }^{5} \mathrm{~A}$ hypercoagulable thromboelastometry profile was defined as at least one of ROTEM assays yielding significantly higher MCF values versus healthy controls. ${ }^{4,6}$

Among 89 consecutive eligible patients, 25 were excluded and 64 finally enrolled (- Supplementary Fig. $\mathbf{~ s 1 , ~ a v a i l a b l e ~ i n ~}$ the online version).

Details on statistical analysis and sample size calculation are reported in -Supplementary Material S1 (available in the online version).

No differences in demographic characteristics were observed between ICU and IMW patients (-Table 1). Sequential Organ Failure Assessment score, incidence of septic shock, and the rate of infection by other respiratory pathogens were significantly higher in ICU patients. Similarly, white blood cells, neutrophils, fibrinogen, C-reactive protein, procalcitonin, ferritin, and fibrinogen levels were significantly higher (-Table $\mathbf{1}$ ).

About ROTEM parameters, MCF values in FIBTEM were significantly higher in ICU than in IMW patients; while CT and CFT in EXTEM were longer and TDI lower in more critically ill patients ( - Table $\mathbf{2}$ ). (c) 2020 Georg Thieme Verlag KG Stuttgart · New York
DOI https://doi.org/ 10.1055/s-0040-1714350. ISSN 0340-6245. 
Table 1 Baseline characteristics, laboratory data, respiratory support, and clinical outcomes

\begin{tabular}{|c|c|c|c|c|c|c|}
\hline Variables & $\begin{array}{l}\mathrm{ICU} \\
n=32\end{array}$ & $\begin{array}{l}\text { IMW } \\
n=32\end{array}$ & $p$-Value & $\begin{array}{l}\mathrm{IMV} \\
n=21\end{array}$ & $\begin{array}{l}\text { Non-IMV } \\
n=43\end{array}$ & $p$-Value \\
\hline \multicolumn{7}{|l|}{ Clinical characteristics } \\
\hline Age $(y)$ & $68(62-75)$ & $61(53-71)$ & 0.08 & $67(63-72)$ & $62(52-75)$ & 0.13 \\
\hline Gender (male) & $26(81)$ & $24(75)$ & 0.76 & $18(86)$ & $32(74)$ & 0.56 \\
\hline BMI & $29(27-32)$ & $29(24-32)$ & 0.94 & $29(27-32)$ & $29(25-32)$ & 0.40 \\
\hline Onset of symptoms (d) & $8(7-11)$ & $8(6-10)$ & 0.56 & 7 (5-9) & $8(7-11)$ & 0.04 \\
\hline Comorbidities & $28(87)$ & $24(75)$ & 0.09 & $21(100)$ & $31(72)$ & $<0.01$ \\
\hline SOFA score & $3(3-6)$ & $2(1-2)$ & $<0.001$ & $4.5(3-7)$ & $2(1-3)$ & $<0.001$ \\
\hline $\begin{array}{l}\text { Sepsis-3 criteria } \\
\text { (septic shock) }\end{array}$ & $9(28)$ & $0(0)$ & $<0.01$ & $8(38)$ & $1(4)$ & $<0.01$ \\
\hline $\begin{array}{l}\text { Other respiratory } \\
\text { pathogens }\end{array}$ & $9(28)$ & $1(3)$ & 0.01 & $8(38)$ & $2(5)$ & $<0.01$ \\
\hline ISTH score & $1(0-2)$ & $0(0-1.8)$ & 0.06 & $1(0-2)$ & $0(0-2)$ & 0.59 \\
\hline SIC score & $2(2-2)$ & $2(1-2)$ & 0.12 & $2(1-2.25)$ & $2(1.8-2)$ & 0.09 \\
\hline $\mathrm{PaO}_{2} / \mathrm{FiO}_{2}$ & $134(121-203)$ & $293(186-354)$ & $<0.001$ & $138(127-220)$ & $226(135-310)$ & 0.02 \\
\hline \multicolumn{7}{|l|}{ Laboratory data } \\
\hline WBCs $\left(\times 10^{9} / \mathrm{L}\right)$ & $8.3(6.0-10.4)$ & $6.7(4.8-8.0)$ & 0.02 & $8.4(6.1-10.9)$ & $6.8(4.9-8.4)$ & 0.06 \\
\hline Neutrophils $\left(\times 10^{9} / \mathrm{L}\right)$ & $7.4(4.7-9.2)$ & $4.5(2.8-6.6)$ & $<0.001$ & $7.6(5.2-9.8)$ & $4.7(2.9-6.6)$ & $<0.001$ \\
\hline Lymphocytes $\left(\times 10^{9} / \mathrm{L}\right)$ & $0.6(0.4-0.9)$ & $1.2(0.7-1.4)$ & 0.33 & $0.5(0.4-0.8)$ & $1.1(0.8-1.4)$ & 0.77 \\
\hline Hemoglobin $(\mathrm{g} / \mathrm{dL})$ & $12(11-13)$ & $13(11-14)$ & 0.19 & $12.3(10.8-13.3)$ & $12.5(11.3-13.9)$ & 0.49 \\
\hline Platelet count $\left(\times 10^{9} / \mathrm{L}\right)$ & $283(194-336)$ & $234(197-290)$ & 0.13 & $225(156-255)$ & $288(202-334)$ & 0.12 \\
\hline PT (\%) & 93 (83-99) & $92(80-101)$ & 0.60 & $95(88-101)$ & 91 (81-99) & 0.12 \\
\hline INR & $1.09(1.06-1.14)$ & $1.09(1.05-1.15)$ & 0.74 & $1.07(1.04-1.12)$ & $1.1(1.05-1.15)$ & 0.48 \\
\hline aPTT (s) & $23(21-27)$ & $25(22-30)$ & 0.43 & $23(21-30)$ & $24(22-29)$ & 0.49 \\
\hline D-dimer (ng/mL) & $315(164-1326)$ & $263(193-598)$ & 0.22 & $277(153-1,059)$ & $263(191-736)$ & 0.52 \\
\hline Fibrinogen $(\mathrm{mg} / \mathrm{dL})$ & $5(4.5-5.7)$ & $4.5(3.3-5.3)$ & 0.04 & $4.8(4.5-5.4)$ & $5.0(3.8-5.4)$ & 0.35 \\
\hline Antithrombin (\%) & $99(91-111)$ & $98(86-104)$ & 0.98 & $98(85-104)$ & $99(90-111)$ & 0.93 \\
\hline $\mathrm{CRP}(\mathrm{mg} / \mathrm{L})$ & $110(55-167)$ & $46(16-96)$ & $<0.001$ & $93(47-165)$ & $64(24-130)$ & 0.12 \\
\hline PCT (ng/mL) & $0.21(0.1-0.99)$ & $0.07(0.04-0.13)$ & $<0.001$ & $0.5(0.07-1.1)$ & $0.09(0.04-0.16)$ & $<0.01$ \\
\hline Interleukin-6 (ug/mL) & $63(22-119)$ & $49(36-109)$ & 0.56 & $85(22-562)$ & $43(23-99)$ & 0.38 \\
\hline Ferritin $(\mathrm{ng} / \mathrm{mL})$ & $1,960(1,250-2,498)$ & $921(610-1,315)$ & $<0.001$ & $1,683(1,148-3,259)$ & $994(647-1,943)$ & 0.01 \\
\hline \multicolumn{7}{|l|}{ Respiratory support } \\
\hline $\mathrm{O}_{2}$-therapy & $0(0)$ & $25(78.1)$ & $<0.001$ & - & - & - \\
\hline HFNC & $0(0)$ & $5(15.6)$ & 0.06 & - & - & - \\
\hline NIV & $11(34.4)$ & $2(6.3)$ & 0.01 & - & - & - \\
\hline IMV & 21 (65.6) & $0(0)$ & $<0.001$ & - & - & - \\
\hline \multicolumn{7}{|l|}{ Outcomes } \\
\hline Symptomatic VTE & $11(34)$ & $3(9)$ & 0.03 & $7(33)$ & $7(16)$ & 0.24 \\
\hline LOHS (d) & $21(16-27)$ & $14(8-18)$ & $<0.001$ & $24(16-34)$ & $15(10-19)$ & $<0.001$ \\
\hline 28-d mortality & $8(25)$ & $1(3)$ & 0.03 & $5(24)$ & $4(9)$ & 0.14 \\
\hline
\end{tabular}

Abbreviations: aPTT, activated partial thromboplastin time; BMI, body mass index; CRP, C-reactive protein; HFNC, high flow nasal cannula; ICU, intensive care unit; IMV, invasive mechanical ventilation; IMW, internal medicine wards; INR, international normalized ratio; ISTH, International Society on Thrombosis and Haemostasis; LOHS, length of hospital stay; NIV, noninvasive ventilation; non-IMV, patients not requiring invasive mechanical ventilation; PCT, procalcitonin; PT, prothrombin time; SIC, sepsis-induced coagulopathy; SOFA, Sequential Organ Failure Assessment; VTE, venous thromboembolisms; WBCs, white blood cells.

Note: Variables are expressed as median and interquartile range (IQR) or number (\%).

Based on the receiver operating characteristic analysis, international normalized ratio, prothrombin time, and activated partial thromboplastin time were strongly predictive of VTE among IMW patients ( - Supplementary Table $\mathbf{S 1}$ and -Supplementary Fig. S2, available in the online version). On the contrary, lymphocytes and platelet count were highly predictive of ICU 28-day mortality (- Supplementary Table $\mathbf{5 2}$ and $\mathbf{- S u p p l e m e n t a r y ~ F i g . ~} \mathbf{5 3}$, available in the online version).

Incidence of VTE, 28-day mortality, and length of hospital stay (LOHS) were significantly higher in ICU patients (-Table 1). 
Table 2 ROTEM parameters

\begin{tabular}{|c|l|l|l|l|l|l|}
\hline Variables & $\begin{array}{l}\text { ICU } \\
\boldsymbol{n}=\mathbf{3 2}\end{array}$ & $\begin{array}{l}\text { IMW } \\
\boldsymbol{n}=\mathbf{3 2}\end{array}$ & $\begin{array}{l}\boldsymbol{p} \text {-Value } \\
\text { IMV } \\
\boldsymbol{n}=\mathbf{2 1}\end{array}$ & $\begin{array}{l}\text { Non-IMV } \\
\boldsymbol{n}=\mathbf{4 3}\end{array}$ & $\boldsymbol{p}$ \\
\hline MCF $(\mathrm{mm})$ & & & & & & \\
\hline EXTEM & $71[65-75]$ & $72[68-75]$ & 0.60 & $69[65-74]$ & $73[69-76]$ & 0.05 \\
\hline INTEM & $68[65-74]$ & $69[65-72]$ & 0.58 & $67[64-72]$ & $70[66-74]$ & 0.34 \\
\hline FIBTEM & $33[27-41]$ & $30[25-33]$ & $\mathbf{0 . 0 2}$ & $29[26-36]$ & $31[26-35]$ & 0.67 \\
\hline CT (s) & & & & & & \\
\hline EXTEM & $74[64-88]$ & $65[61-72]$ & $<\mathbf{0 . 0 1}$ & $74[65-87]$ & $66[61-78]$ & 0.06 \\
\hline INTEM & $184[159-203]$ & $174[162-182]$ & 0.22 & $187[158-205]$ & $174[162-184]$ & 0.23 \\
\hline CFT (s) & & & & & & \\
\hline EXTEM & $60[48-80]$ & $43[38-56]$ & $<\mathbf{0 . 0 1}$ & $61[51-83]$ & $45[38-58]$ & $<0.01$ \\
\hline INTEM & $55[46-64]$ & $47[40-61]$ & 0.10 & $57[48-64]$ & $47[40-59]$ & 0.10 \\
\hline TDI & & & & & & \\
\hline EXTEM & $0.55[0.48-0.62]$ & $0.65[0.54-0.72]$ & $\mathbf{0 . 0 2}$ & $0.57[0.46-0.62]$ & $0.63[0.52-0.72]$ & $\mathbf{0 . 0 2}$ \\
\hline INTEM & $0.32[0.24-0.35]$ & $0.31[0.25-0.35]$ & 0.92 & $0.29[0.24-0.34]$ & $0.32[0.27-0.35]$ & 0.43 \\
\hline
\end{tabular}

Abbreviations: CFT, clot formation time; CT, clotting time; ICU, intensive care unit; IMV, invasive mechanical ventilation; IMW, internal medicine wards; MCF, maximum clot firmness; non-IMV, patients not requiring invasive mechanical ventilation; TDI, ThromboDynamic Index. Note: Variables are expressed as median and interquartile range (IQR).

Twenty-one (66\%) patients required invasive mechanical ventilation and showed rapid onset of symptoms and longer LOHS (-Table 1). In these patients, CFT was prolonged and TDI in EXTEM was lower, while neutrophils and ferritin levels were significantly higher. No differences in MCF values were observed ( - Table $\mathbf{2}$ ).

Our study showed that COVID-19 patients with mild respiratory failure admitted to IMW had less severe hypercoagulability and lower incidence of symptomatic VTE as compared with more critically ill patients. Although traditional risk factors associated with adverse outcome of ICU patients (i.e., immobility, hemodynamic instability, etc.) certainly played a fundamental role, these findings might have contributed, at least in part, to the higher 28-day mortality observed in more critically ill patients.

Interestingly, traditional coagulative parameters, other than fibrinogen, were similar between ICU and IMW patients and no differences in D-dimer levels were found. These surprising findings would seem to be in contrast with those reported by other published studies. ${ }^{1,2}$ Nonetheless, the high standard deviation of D-dimer values that we observed, added to the relatively small sample size, may justify these differences. On the contrary, IMW patients presented with lower FIBTEM-MCF values as reflected in part by the higher fibrinogen levels observed in ICU patients. FIBTEM test, which is a better indicator of fibrin formation/polymerization and fibrinogen concentration during the acute phase, was maximally expressed in ICU patients. Notably, EXTEM-CT and EXTEM-CFT were slightly shorter and TDI higher in IMW patients, probably due to vitamin $\mathrm{K}$ deficiency and impaired liver synthesis and carboxylation of coagulation factors, frequently observed in ICU patients. $^{7}$

Our results showed that neither ICU nor IMW patients presented with consumptive coagulopathy (e.g., disseminat- ed intravascular coagulation) often related to the presence of significantly elevated D-dimer levels, reduction of platelet count, prolongation of CTs, antithrombin reduction, or fibrinogen consumption. ${ }^{4,8,9}$

Our results deserve some comments. Only symptomatic VTE were considered, probably underestimating the real incidence of thrombosis. This is particularly important, since IMW patients received standard thromboprophylaxis with low molecular weight heparin and ICU patients with intermediate dose in agreement with the indication of the treating physician and recent findings. ${ }^{10-12}$ Although some protocols on the use of clinical and analytic parameters, as D-dimer levels, to manage heparin dose were recently published, ${ }^{10-12}$ our data did not allow to assess the impact of adequate dosages of anticoagulants on clotting parameters. Moreover, whether thromboelastometry parameters can be used to monitor the correct dosing of thromboprophylaxis is still a matter of debate.

The heterogeneity between ICU and IMW cohorts represents one of the main limitations to interpret our findings. Furthermore, we acknowledge that several clinical variables might have affected the differences in COVID-19-related hypercoagulable state. Moreover, the sample size, especially in the subgroup of IMW patients, could not be representative of all hospitalized COVID-19 patients. In addition, the use of thromboelastometry to assess hypercoagulability could be questioned because of the lack of standardization which can limit the comparison of data between centers. Moreover, it is well known that thromboelastometry evaluates some aspects of coagulation and clot formation but important information including platelet function or the thrombin generation potential can be missed.

In conclusion, patients with life-threatening COVID-19 infection and hospitalized in ICU showed more severe hypercoagulability, higher FIBTEM-MCF values, and thrombotic 
risk as compared with IMW patients. The assessment of hypercoagulability through conventional coagulation tests and ROTEM could be helpful for the identification of more appropriate thromboprophylaxis strategies. Additional studies on different laboratory tests to extensively evaluate hypercoagulability in COVID-19 patients are needed.

\section{What is known about this topic?}

- Coagulation abnormalities were common findings in critically ill COVID-19 patients.

- COVID-19 patients with acute respiratory failure admitted to intensive care units (ICUs) present with severe hypercoagulability.

\section{What does this paper add?}

- Patients with mild acute respiratory failure secondary to SARS-CoV-2 infection hospitalized in internal medicine ward (IMW) and critically ill patients requiring ICU admission showed severe hypercoagulability.

- ICU patients presented with a more severe COVID-19related hypercoagulability than IMW patients mainly due to higher FIBTEM-MCF values related to fibrinogen levels.

- A higher thrombotic risk was observed in ICU as compared with IMW patients.

\section{Authors' Contributions}

A.B., L.S., P.S., and P.N. designed the study. C.C., N.S., E.C., F.P., L.C., and M.C. collected data. L.P., A.D.C., E.P., and L.B. analyzed the data. A.B. and L.S. equally contributed to write the manuscript. P.S., P.N., E.S., A.M.C., and R.V. edited the manuscript.

\section{Conflict of Interest}

None declared.

\section{References}

1 Lippi G, Favaloro EJ. D-dimer is associated with severity of coronavirus disease 2019: a pooled analysis. Thromb Haemost 2020;120(05):876-878

2 Tang N, Li D, Wang X, Sun Z. Abnormal coagulation parameters are associated with poor prognosis in patients with novel coronavirus pneumonia. J Thromb Haemost 2020;18(04):844-847

3 Han H, Yang L, Liu R, et al. Prominent changes in blood coagulation of patients with SARS-CoV-2 infection. Clin Chem Lab Med 2020; 58(07):1116-1120

4 Spiezia L, Boscolo A, Poletto F, et al. COVID-19 -related severe hypercoagulability in patients admitted to intensive care unit for acute respiratory failure. Thromb Haemost 2020;120(06):998-1000

5 Boscolo A, Spiezia L, Campello E, Consolaro EM, Ori C, Simioni P. The prognostic role of ThromboDynamic Index in patients with severe sepsis. Intern Emerg Med 2020;15(01):163-168

6 Spiezia L, Radu C, Marchioro P, et al. Peculiar whole blood rotation thromboelastometry (ROTEM) profile in 40 sideropenic anaemia patients. Thromb Haemost 2008;100(06):1106-1110

7 Mercer KW, Gail Macik B, Williams ME. Hematologic disorders in critically ill patients. Semin Respir Crit Care Med 2006;27(03): 286-296

8 Ranucci M, Ballotta A, Di Dedda U, et al. The procoagulant pattern of patients with COVID-19 acute respiratory distress syndrome. J Thromb Haemost 2020;18(07):1747-1751

9 Panigada M, Bottino N, Tagliabue P, et al. Hypercoagulability of COVID-19 patients in intensive care unit. a report of thromboelastography findings and other parameters of hemostasis. J Thromb Haemost 2020;18(07):1738-1742

10 Bikdeli B, Madhavan MV, Jimenez D, et al; Global COVID-19 Thrombosis Collaborative Group, Endorsed by the ISTH, NATF, ESVM, and the IUA, Supported by the ESC Working Group on Pulmonary Circulation and Right Ventricular Function. COVID-19 and thrombotic or thromboembolic disease: implications for prevention, antithrombotic therapy, and follow-up: JACC Stateof-the-Art Review. J Am Coll Cardiol 2020;75(23):2950-2973

11 Vivas D, Roldán V, Esteve-Pastor MA, et al; Posicionamiento del Grupo de Trabajo de Trombosis Cardiovascular de la Sociedad Española de Cardiología. Recomendaciones sobre el tratamiento antitrombótico durante la pandemia COVID-19. Rev Esp Cardiol 2020;73(09):749-757

12 Tang N, Bai H, Chen X, et al. Anticoagulant treatment is associated with decreased mortality in severe coronavirus disease 2019 patients with coagulopathy. J Thromb Haemost 2020;19(04): 844-847 